\title{
Spin of ground state baryons
}

\author{
A. J. Buchmann ${ }^{1}$, E. M. Henley ${ }^{2}$ \\ Institut für Theoretische Physik, Universität Tübingen \\ Auf der Morgenstelle 14, D-72076 Tübingen, Germany \\ 2 Department of Physics and Institute for Nuclear Theory, Box 351560, \\ University of Washington, Seattle, WA 98195, U.S.A.
}

\begin{abstract}
We calculate the quark spin contribution to the total angular momentum of flavor octet and flavor decuplet ground state baryons using a spin-flavor symmetry based parametrization method of quantum chromodynamics. We find that third order SU(6) symmetry breaking three-quark operators are necessary to explain the experimental result $\Sigma_{1}=0.32(10)$. For spin $3 / 2$ decuplet baryons we predict that the quark spin contribution is $\Sigma_{3}=3.93(22)$, i.e. considerably larger than their total angular momentum.
\end{abstract}

PACS numbers: 11.30.Ly, 12.38.Lg, 14.20.-c 


\section{INTRODUCTION}

The question how the proton spin is made up from the quark spin $\Sigma$, quark orbital angular momentum $L_{q}$, gluon spin $\Delta g$, and gluon orbital angular momentum $L_{g}$

$$
J=\frac{1}{2} \Sigma+L_{q}+\Delta g+L_{g}
$$

is one of the central issues in nucleon structure physics [1, 2]. In the constituent quark model with only one-body operators one obtains $J=\Sigma / 2=1 / 2$, i.e., the proton spin is the sum of the constituent quark spins and nothing else. However, experimentally it is known that only about $1 / 3$ of the proton spin comes from quarks [3, 4]. The disagreement between the quark model result and experiment came as a surprise because the same model accurately described the related proton and neutron magnetic moments.

Using a broken SU(6) spin-flavor symmetry based parametrization of quantum chromodynamics we show that the failure of the quark model to describe the quark contribution to proton spin correctly is due to the neglect of three-quark terms in the axial current. Including third order SU(6) symmetry breaking three-quark terms we reproduce the measured quark contribution to the proton spin and predict the spin carried by quarks for the remaining flavor octet and decuplet ground state baryons.

\section{SPIN-FLAVOR SYMMETRY AND QCD PARAMETRIZATION METHOD}

We use a general parametrization method developed by Morpurgo [5] to calculate the quark contribution to baryon spin in a systematic manner. This method is based on broken spin-flavor symmetry and quark-gluon dynamics of quantum chromodynamics (QCD). The basic idea is to formally define, for the observable at hand, a QCD operator $\Omega$ and QCD eigenstates $|B\rangle$ expressed explicitly in terms of quarks and gluons. The corresponding matrix elements can, with the help of the unitary operator $V$, be reduced to an evaluation in a basis of pure three-quark states $\left|\Phi_{B}\right\rangle$ with orbital angular momentum $L=0$

$$
\langle B|\Omega| B\rangle=\left\langle\Phi_{B}\left|V^{\dagger} \Omega V\right| \Phi_{B}\right\rangle=\left\langle W_{B}|\tilde{\Omega}| W_{B}\right\rangle
$$

The spin-flavor wave functions contained in $\left|\Phi_{B}\right\rangle$ are denoted by $\left|W_{B}\right\rangle$. The operator $V$ dresses the pure three-quark states with $q \bar{q}$ components and gluons and thereby generates the exact QCD eigenstates $|B\rangle$. 
The main task is to find the most general expression for the operator $\tilde{\Omega}$ that is compatible with the space-time and inner QCD symmetries. Usually, this is a sum of one-, two-, and three-quark operators in spin-flavor space multiplied by a priori unknown constants which parametrize the orbital and color space matrix elements. Empirically, a hierarchy in the importance of one-, two-, and three-quark operators is found. This fact can be understood in the $1 / N_{c}$ expansion of QCD where two- and three-quark operators describing second and third order $\mathrm{SU}(6)$ symmetry breaking are usually suppressed by powers of $1 / N_{c}$ and $1 / N_{c}^{2}$ respectively compared to one-quark operators associated with first order symmetry breaking [6]. Previously, we have applied this method also to calculate higher order corrections to baryon-meson couplings as well as baryon electromagnetic moments [7].

To begin with, we show which spin-flavor structures contribute to the flavor singlet axial current. Generally, an SU(6) spin-flavor symmetry breaking operator $\tilde{\Omega}^{R}$ acting on the $\mathbf{5 6}$ dimensional baryon ground state supermultiplet must transform according to one of the irreducible representations $R$ contained in the direct product [8]

$$
56 \times 56=1+35+405+2695 .
$$

The 1 dimensional representation (rep) on the right-hand side of Eq.(2.2) corresponds to an $\mathrm{SU}(6)$ symmetric operator, while the 35, 405, and 2695 dimensional reps characterize respectively, first, second, and third order SU(6) symmetry breaking. Therefore, a general $\mathrm{SU}(6)$ breaking operator has the form

$$
\tilde{\Omega}=\tilde{\Omega}^{1}+\tilde{\Omega}^{35}+\tilde{\Omega}^{405}+\tilde{\Omega}^{2695} .
$$

In terms of quarks, the operators on the right-hand side of Eq.(2.3) are represented respectively by zero-, one-, two-, and three-quark operators [9]. The two- and three-quark operators are an effective description of quark-antiquark and gluon degrees of freedom that have been moved from the QCD eigenstates $|B\rangle$ to the operator $\tilde{\Omega}$ by virtue of the unitary transformation $V$.

We now decompose each SU(6) tensor $\tilde{\Omega}^{R}$ into subtensors $\tilde{\Omega}_{(F, 2 J+1)}^{R}$ with definite transformation properties with respect to the $\mathrm{SU}(3)_{F}$ flavor and $\mathrm{SU}(2)_{J}$ spin subgroups of $\mathrm{SU}(6)$. To describe spin, flavor singlet, axial vector subtensors of type $\tilde{\Omega}_{(1,3)}^{R}$ are required. Denoting operators by their dimensionalities for simplicity we obtain [10, 11]

$$
35=(8,3)+(8,1)+(1,3),
$$




$$
\begin{aligned}
\mathbf{4 0 5} & =(\mathbf{2 7}, \mathbf{5})+(\mathbf{8}, \mathbf{5})+(\mathbf{1}, \mathbf{5})+(\mathbf{2 7}, \mathbf{3})+(\mathbf{1 0}, \mathbf{3})+(\overline{\mathbf{1 0}}, \mathbf{3})+2(\mathbf{8}, \mathbf{3}) \\
& +(\mathbf{2 7}, \mathbf{1})+(\mathbf{8}, \mathbf{1})+(\mathbf{1}, \mathbf{1}) \\
\mathbf{2 6 9 5} & =(\mathbf{6 4}, \mathbf{7})+(\mathbf{2 7}, \mathbf{7})+(\mathbf{8}, \mathbf{7})+(\mathbf{1}, \mathbf{7}) \\
& +(\mathbf{6 4}, \mathbf{5})+(\mathbf{3 5}, \mathbf{5})+(\overline{\mathbf{3 5}, 5})+2(\mathbf{2 7}, \mathbf{5})+(\mathbf{1 0}, \mathbf{5})+(\overline{\mathbf{1 0}}, \mathbf{5})+2(\mathbf{8}, \mathbf{5}) \\
& +(\mathbf{6 4}, \mathbf{3})+(\mathbf{3 5}, \mathbf{3})+(\overline{\mathbf{3 5}}, \mathbf{3})+3(\mathbf{2 7}, \mathbf{3})+2(\mathbf{1 0}, \mathbf{3})+2(\mathbf{8}, \mathbf{3})+(\mathbf{1}, \mathbf{3}) \\
& +(\mathbf{6 4}, \mathbf{1})+(\mathbf{2 7}, \mathbf{1})+(\mathbf{1 0}, \mathbf{1})+(\overline{\mathbf{1 0}}, \mathbf{1})+(\mathbf{8}, \mathbf{1}) .
\end{aligned}
$$

Here, the first and second entries in the parentheses refer to the dimensions of the $\mathrm{SU}(3)_{F}$ and $\mathrm{SU}(2)_{J}$ reps according to which the corresponding operators transform.

From the flavor-spin decompositions in Eq.(2.4) it is clear that a flavor singlet axial vector operator $(\mathbf{1}, \mathbf{3})$ needed to describe baryon spin, is contained only in the $\mathbf{3 5}$ and $\mathbf{2 6 9 5}$

dimensional reps of $\mathrm{SU}(6)$ so that we can write $\mathbf{A}:=\tilde{\Omega}=\tilde{\Omega}_{(\mathbf{1}, \mathbf{3})}^{35}+\tilde{\Omega}_{(\mathbf{1}, \mathbf{3})}^{2695}$. As a result, the flavor singlet axial vector current contains only a one-quark and a three-quark term

$$
\mathbf{A}=\mathbf{A}_{[1]}+\mathbf{A}_{[3]}=A \sum_{i=1}^{3} \boldsymbol{\sigma}_{i}+C \sum_{i \neq j \neq k}^{3} \boldsymbol{\sigma}_{i} \cdot \boldsymbol{\sigma}_{j} \boldsymbol{\sigma}_{k},
$$

where $\boldsymbol{\sigma}_{i}$ is the Pauli spin matrix of quark $i$ and the constants $A$ and $C$ are to be determined from experiment. Two-quark operators, such as $\sum_{i \neq j} \boldsymbol{\sigma}_{i} \times \boldsymbol{\sigma}_{j}$ or $\sum_{i \neq j}\left(\boldsymbol{\sigma}_{i} \cdot \boldsymbol{\sigma}_{j}\right) \boldsymbol{\sigma}_{i}$ add up to zero or can be reduced to one-body operators. Therefore, there is no two-quark contribution to the flavor singlet axial vector current $\mathbf{A}$, in agreement with the general symmetry argument that the 405 rep does not contain a $(\mathbf{1}, \mathbf{3})$ operator structure.

In Ref. [12] a flavor singlet two-body gluon exchange current $\mathbf{A}_{0}$ was constructed from the flavor octet axial axial current $\mathbf{A}_{8}$ by replacing the Gell-Mann matrix $\boldsymbol{\lambda}_{8}$ with the SU(3) flavor singlet matrix $\boldsymbol{\lambda}_{0}$. From the perspective of broken SU(6) symmetry, such a two-body exchange current, which has also been used in Ref. [13], does not exist.

\section{SPIN-FLAVOR WAVE FUNCTIONS}

The SU(6) spin-flavor wave functions of octet baryons are, for example, for the proton in standard notation

$$
\begin{aligned}
|p \uparrow\rangle=\frac{1}{\sqrt{2}} & \left\{\frac{1}{\sqrt{6}}|(2 u u d-u d u-d u u)\rangle \frac{1}{\sqrt{6}}|(2 \uparrow \uparrow \downarrow-\uparrow \downarrow \uparrow-\downarrow \uparrow \uparrow)\rangle\right. \\
& \left.+\frac{1}{\sqrt{2}}|(u d u-d u u)\rangle \frac{1}{\sqrt{2}}|(\uparrow \downarrow \uparrow-\downarrow \uparrow \uparrow)\rangle\right\} .
\end{aligned}
$$


Alternatively, one can write this wave function as

$$
\begin{aligned}
& |p \uparrow\rangle=\frac{1}{\sqrt{18}} \quad \mid 2 u \uparrow u \uparrow d \downarrow-u \uparrow u \downarrow d \uparrow-u \downarrow u \uparrow d \uparrow \\
& +2 d \downarrow u \uparrow u \uparrow-d \uparrow u \downarrow u \uparrow-d \uparrow u \uparrow u \downarrow \\
& +2 u \uparrow d \downarrow u \uparrow-u \uparrow d \uparrow u \downarrow-u \downarrow d \uparrow u \uparrow\rangle \text {. }
\end{aligned}
$$

Likewise one can write down the spin-flavor wave functions of other octet and decuplet baryons [14].

\section{QUARK SPIN CONTRIBUTION TO BARYON SPIN}

The matrix elements of the quark contribution to baryon spin can be straightforwardly

calculated by sandwiching the flavor singlet axial current A of Eq.(2.5) between SU(6) baryon wave functions, e.g. for the proton in Eq.(3.2). Our results for the spin 1/2 octet and the spin $3 / 2$ decuplet baryons are

$$
\begin{aligned}
& \Sigma_{1}:=\left\langle B_{8} \uparrow\left|\mathbf{A}_{z}\right| B_{8} \uparrow\right\rangle=A-10 C \\
& \Sigma_{3}:=\left\langle B_{10} \uparrow\left|\mathbf{A}_{z}\right| B_{10} \uparrow\right\rangle=3 A+6 C,
\end{aligned}
$$

where $B_{8}\left(B_{10}\right)$ stands for any member of the baryon flavor octet (decuplet). Here, $\Sigma_{i}$ is twice the quark spin contribution to the total baryon angular momentum. We predict the same quark contribution to the total baryon angular momentum for all members of a given flavor multiplet independent of the flavor composition of individual baryons. This is to be expected because we are dealing with a flavor singlet operator that does not break $\mathrm{SU}(3)$ symmetry. On the other hand, we find that $\mathrm{SU}(6)$ symmetry is broken as reflected by the different expressions for flavor octet and decuplet baryons.

To calculate the contribution of individual quark flavors we define one-body $u$-quark and $d$-quark operators acting only on $u$-quarks and $d$-quarks as

$$
\mathbf{A}_{[1] z}^{u}=A \sum_{i=1}^{3} \boldsymbol{\sigma}_{i z}^{u}, \quad \mathbf{A}_{[1] z}^{d}=A \sum_{i=1}^{3} \boldsymbol{\sigma}_{i z}^{d},
$$

with matrix elements between proton wave functions

$$
\left\langle p \uparrow\left|A \sum_{i=1}^{3} \boldsymbol{\sigma}_{i z}^{u}\right| p \uparrow\right\rangle=\frac{4}{3} A, \quad\left\langle p \uparrow\left|A \sum_{i=1}^{3} \boldsymbol{\sigma}_{i z}^{d}\right| p \uparrow\right\rangle=-\frac{1}{3} A .
$$


Adding both contributions gives

$$
\left\langle p \uparrow\left|A \sum_{i=1}^{3}\left(\boldsymbol{\sigma}_{i z}^{u}+\boldsymbol{\sigma}_{i z}^{d}\right)\right| p \uparrow\right\rangle=\frac{4}{3} A-\frac{1}{3} A=A,
$$

in agreement with the first term of Eq.(4.1).

Analogously, three-body $u$-quark and $d$-quark operators are defined as

$$
\begin{aligned}
& \mathbf{A}_{[3] z}^{u}=2 C\left(\boldsymbol{\sigma}_{1}^{u} \cdot \boldsymbol{\sigma}_{2}^{d} \boldsymbol{\sigma}_{3 z}^{u}+\boldsymbol{\sigma}_{1}^{d} \cdot \boldsymbol{\sigma}_{2}^{u} \boldsymbol{\sigma}_{3 z}^{u}+\boldsymbol{\sigma}_{1}^{u} \cdot \boldsymbol{\sigma}_{3}^{d} \boldsymbol{\sigma}_{2 z}^{u}+\boldsymbol{\sigma}_{1}^{d} \cdot \boldsymbol{\sigma}_{3}^{u} \boldsymbol{\sigma}_{2 z}^{u}+\boldsymbol{\sigma}_{2}^{u} \cdot \boldsymbol{\sigma}_{3}^{d} \boldsymbol{\sigma}_{1 z}^{u}+\boldsymbol{\sigma}_{2}^{d} \cdot \boldsymbol{\sigma}_{3}^{u} \boldsymbol{\sigma}_{1 z}^{u}\right), \\
& \mathbf{A}_{[3] z}^{d}=C\left(\boldsymbol{\sigma}_{1}^{u} \cdot \boldsymbol{\sigma}_{2}^{u} \boldsymbol{\sigma}_{3 z}^{d}+\boldsymbol{\sigma}_{2}^{u} \cdot \boldsymbol{\sigma}_{1}^{u} \boldsymbol{\sigma}_{3 z}^{d}+\boldsymbol{\sigma}_{1}^{u} \cdot \boldsymbol{\sigma}_{3}^{u} \boldsymbol{\sigma}_{2 z}^{d}+\boldsymbol{\sigma}_{3}^{u} \cdot \boldsymbol{\sigma}_{1}^{u} \boldsymbol{\sigma}_{2 z}^{d}+\boldsymbol{\sigma}_{2}^{u} \cdot \boldsymbol{\sigma}_{3}^{u} \boldsymbol{\sigma}_{1 z}^{d}+\boldsymbol{\sigma}_{3}^{u} \cdot \boldsymbol{\sigma}_{2}^{u} \boldsymbol{\sigma}_{1 z}^{d}\right),
\end{aligned}
$$

with matrix elements

$$
\left\langle p \uparrow\left|\mathbf{A}_{[3] z}^{u}\right| p \uparrow\right\rangle=-\frac{28}{3} C, \quad\left\langle p \uparrow\left|\mathbf{A}_{[3] z}^{d}\right| p \uparrow\right\rangle=-\frac{2}{3} C .
$$

The total three-quark spin contribution to proton spin is

$$
\left\langle p \uparrow\left|\mathbf{A}_{[3] z}^{u}+\mathbf{A}_{[3] z}^{d}\right| p \uparrow\right\rangle=-\frac{28}{3} C-\frac{2}{3} C=-10 C,
$$

as it should be according to Eq.(4.1). Summarizing, we obtain for the $u$ - and $d$-quark contributions to the spin of the proton

$$
\begin{aligned}
& \Delta u:=\left\langle p \uparrow\left|\mathbf{A}_{[1] z}^{u}+\mathbf{A}_{[3] z}^{u}\right| p \uparrow\right\rangle=\frac{4}{3} A-\frac{28}{3} C \\
& \Delta d:=\left\langle p \uparrow\left|\mathbf{A}_{[1] z}^{d}+\mathbf{A}_{[3] z}^{d}\right| p \uparrow\right\rangle=-\frac{1}{3} A-\frac{2}{3} C .
\end{aligned}
$$

We fix the constants $A$ and $C$ as follows. The combined deep inelastic scattering and hyperon $\beta$-decay data give [4]

$$
\Delta u=0.83 \pm 0.03, \quad \Delta d=-0.43 \pm 0.03, \quad \Delta s=-0.09 \pm 0.04
$$

The sum of these experimental spin fractions $\Sigma_{1_{\text {exp }}}=\Delta u+\Delta d+\Delta s=0.32(10)$ is considerably smaller than expected from the additive quark model, which gives $\Sigma_{1}=1$. Solving Eq.(4.8) for $A$ and $C$ we get

$$
\begin{aligned}
& A=\frac{1}{6} \Delta u-\frac{7}{3} \Delta d \\
& C=-\frac{1}{12} \Delta u-\frac{1}{3} \Delta d .
\end{aligned}
$$


Inserting the experimental results for $\Delta u$ and $\Delta d$ from Eq.(4.9) we obtain $A=1.15(7)$ and $C=0.08(1)$.

According to Eq.(4.1) the quark spin contribution to the total angular momentum of flavor octet baryons is then $\Sigma_{1}=0.35(12)$ compared to the experimental result $\Sigma_{1_{\text {exp }}}=0.32(10)$. Despite the typical $1 / N_{c}^{2} \cong 1 / 9$ suppression of three-quark compared to one-quark terms, for octet baryon spin, the three-quark contribution is of the same importance as the onequark term because of the factor 10 multiplying the $C$ term. As a result of the cancellation between the one-quark and the three-quark terms one finds that quark spin contributes only $1 / 3$ of the total octet baryon spin. As mentioned before, the three-quark term is an effective description of quark-antiquark and gluon degrees of freedom in physical baryons. These degrees of freedom are also responsible for orbital angular momentum that is needed to obtain the total baryon spin.

With $A$ and $C$ fixed, we predict the quark spin contribution to decuplet baryon spin not considered before by other authors as

$$
\Sigma_{3}=\left\langle B_{10} \uparrow|\mathbf{A}| B_{10} \uparrow\right\rangle=3 A+6 C=3.93(22) .
$$

It is interesting that for decuplet baryons, quark spins add up to 1.31 times the additive quark model value $2 S_{z}=3$. Therefore, while orbital angular momentum must provide a positive contribution for octet baryons, it must reduce the quark spin in the case of decuplet baryons.

\section{SUMMARY}

In summary, we have presented a straightforward calculation of the quark spin contribution to the total angular momentum of ground state baryons using a spin-flavor symmetry based parametrization of QCD. For flavor octet baryons, we have shown that three-quark operators modify the standard quark model prediction based on one-quark operators from $\Sigma_{1}=1$ to $\Sigma_{1}=0.35(12)$ in agreement with the experimental result. On the other hand, in the case of flavor decuplet baryons, three-quark operators enhance the contribution of one quark operators from $\Sigma_{3}=3$ to $\Sigma_{3}=3.93(22)$.

In this paper, our concern has been with the quark spin contribution to baryon total

angular momentum. There are two further contributions, the orbital angular momentum of 
the quarks and the gluon contributions. Concerning gluon spin $\Delta g$, different experiments agree that it is too small to explain the total proton angular momentum [15]. Furthermore, it has been shown that gluon orbital angular momentum $L_{g}$ is very small as well [16]. Quark

orbital angular momentum $L_{q}$ has recently been investigated by a number of authors [17-19]. We do not attempt to discuss these contributions here, because it would detract from our main theme. We hope to address this issue in a future publication.

Acknowledgments: One of the authors (EMH) is grateful to the Humboldt Foundation for a grant and to Wolfram Weise and the Technical University of Munich for their hospitality.

[1] L. M. Sehgal, Phys. Rev. D 10, 1663 (1974).

[2] Xiangdong Ji, Phys. Rev. Lett. 78, 610 (1997).

[3] J. Ashman et al., Phys. Lett. B 206, 364 (1988); Nucl. Phys. B328, 1 (1989).

[4] K. Abe et al. (E143 Collaboration), Phys. Rev. D 58, 112003 (1998).

[5] G. Morpurgo, Phys. Rev. D 40, 2997 (1989) 2997; Phys. Rev. D 40, 3111 (1989).

[6] A. J. Buchmann and R. F. Lebed, Phys. Rev. D 62, 096005 (2000).

[7] A. J. Buchmann, E. M. Henley, Phys. Lett. B 484, 255 (2000); Phys. Rev. C 63, 015202 (2000); Phys. Rev. D 65, 073017 (2002); Eur. Phys. J. A 35, 267 (2008).

[8] B. Sakita, Phys. Rev. Lett. 13, 643 (1964).

[9] R. F. Lebed, Phys. Rev. D 51, 5039 (1995).

[10] M. A. B. Beg, V. Singh, Phys. Rev. Lett. 13, 418 (1964).

[11] C. L. Cook and G. Murtaza, Nuov. Cim 39, 531 (1965).

[12] D. Barquilla-Cano, A. J. Buchmann, E. Hernández, Eur. Phys. J. A 27, 365 (2006).

[13] F. Myhrer, A. W. Thomas, Phys. Lett. B 663, 302 (2008).

[14] D. B. Lichtenberg, Unitary Symmetry and Elementary Particles, Academic Press, N.Y., 1970.

[15] A. W. Thomas, Int. J. Mod. Phys. E18, 1116 (2009).

[16] S. J. Brodsky and S. Gardner, Phys. Lett. B 643, 22 (2006).

[17] A. W. Thomas, Phys. Lett. 101, 102003 (2008).

[18] M. Wakamatsu and Y. Nakakoji, Phys. Rev. D 77, 074011 (2008); M. Wakamatsu, Eur. Phys. J. A 44, 297 (2010)).

[19] G. T. Garvey, Phys. Rev. C 81, 055212 (2010). 